Journal for ImmunoTherapy of Cancer

\section{Narrowband ultraviolet B therapy for refractory immune-related lichenoid dermatitis on PD-1 therapy: a case report}

To cite: Esfahani K, Henderson Berg M-H, Zargham $\mathrm{H}$, et al. Narrowband ultraviolet B therapy for refractory immunerelated lichenoid dermatitis on PD-1 therapy: a case report. Journal for ImmunoTherapy of Cancer 2021;9:e001831. doi:10.1136/jitc-2020-001831

KE and M-HHB contributed equally.

$\mathrm{OR}$ and WHM contributed equally.

$\mathrm{KE}$ and $\mathrm{M}-\mathrm{HHB}$ are joint first authors.

Accepted 10 February 2021

Check for updates

(C) Author(s) (or their employer(s)) 2021. Re-use permitted under CC BY-NC. No commercial re-use. See rights and permissions. Published by BMJ.

${ }^{1}$ Division of Oncology, Department of Medicine, McGill University, Montreal, Quebec, Canada

${ }^{2}$ Division of Dermatology, Department of Medicine, McGill University, Montreal, Quebec,

Canada

${ }^{3}$ Department of Pathology, McGill University, Montreal, Quebec, Canada

Correspondence to Dr Khashayar Esfahani, Department of Medicine, Division of Oncology, Jewish General Hospital, Montreal,

Canada;

khashayar.esfahani@mcgill.ca

\section{ABSTRACT}

Treatment with programmed cell death 1 inhibitors is associated with a wide range of cutaneous immunerelated adverse events, with lichenoid eruptions representing one of the major cutaneous toxicities. We describe the case of an 81-year-old man with metastatic melanoma treated with pembrolizumab who subsequently developed a delayed-onset generalized lichenoid dermatitis. After failing multiple lines of systemic immunosuppression, narrowband ultraviolet B (NBUVB) phototherapy three times per week for 17 sessions resulted in a significant clinical response in his cutaneous eruption and was well tolerated. NBUVB is a safe, lower-cost modality that induces local, skin-specific immunosuppression without the toxicities of traditional systemic immunosuppressive agents. To date, this is the first report of use of NBUVB in immune-related lichenoid dermatitis resistant to multiple standard therapies.

\section{INTRODUCTION}

Immune checkpoint inhibitors (ICIs) directed against T-lymphocyte-associated antigen 4, programmed cell death 1 (PD-1) and programmed cell death ligand 1 (PDL1) have transformed long-term outcomes in advanced melanoma. Their use can come at the expense of a wide range of immunerelated adverse events, of which cutaneous manifestations can account for up to $30 \%$ of the reported toxicities. ${ }^{1}$ A variety of reactions have been described, including morbilliform, lichenoid, eczematous, immune-bullous, psoriasiform eruptions, and vitiligo-like depigmentation. ${ }^{2}$ Although most cases are managed with topical corticosteroids and/ or oral corticosteroids, a subset of patients will be refractory to this first line of therapy and require systemic immunosuppression with other agents. In those cases, no clear standard of care exists, and treatments are often tailored to the clinical phenotype of the adverse reaction, to the findings on histopathology, or to similar treatments used in the primary forms of the same autoimmune skin reactions. Finding the right therapy can be challenging, as the benefits of systemic immunosuppression must be weighed against the risks of opportunistic infections or the potential blunting of cancer immune surveillance. ${ }^{3}$

Narrowband ultraviolet B (NBUVB) phototherapy is an established, lower cost, and often preferred treatment option for many common skin conditions, both benign and malignant, and carries a relatively benign side-effect profile. NBUVB can induce T-cell anergy and reduce the local production of inflammatory cytokines with minimal systemic toxicity. ${ }^{4}$ Interestingly, although its use has been reported previously, treatment with NBUVB has not yet been described in cutaneous immune-related toxicities that are refractory to multiple standard therapies, where the avoidance of systemic immunosuppression is particularly appealing. Herein, we report a case of a patient on pembrolizumab who developed generalized lichenoid dermatitis refractory to steroids and three lines of systemic immunosuppressive agents (mycophenolate mofetil, methotrexate, and cyclosporine) and who achieved excellent control of his dermatological toxicity with NBUVB phototherapy.

\section{CASE PRESENTATION}

The patient is an 81-year-old man with metastatic BRAF V600R mutated melanoma. He is known for a remote history of mantle cell lymphoma for which he is in remission poststem cell transplantation, mild oral lichen planus, for which he had never required treatment, gout, gastroesophageal reflux disease, chronic bronchitis, benign prostatic hyperplasia, and depression. There is no history of hepatitis $\mathrm{C}$ infection, renal disease, or diabetes mellitus. After failing a firstline course of BRAF-targeted therapy with dabrafenib and trametinib, he started with second-line pembrolizumab in November of 
2018. In May of 2019, pembrolizumab was discontinued due to the serial progression of disease on imaging. Within 6 weeks of discontinuation of therapy, the patient presented with a generalized skin eruption, composed of multiple scaly erythematous papules and plaques, some with central hyperkeratotic crust, in a symmetric distribution on the arms, chest, back, abdomen, and upper legs. No other medications were introduced prior to development of the cutaneous eruption (figure 1). A skin biopsy was performed on his right arm to evaluate the lesions. Histopathology revealed a lichenoid interface dermatitis with typical histological features of a lichenoid drug reaction, such as eosinophils and the presence of dyskeratotic cells (colloid bodies) above the basal layer of the epidermis (figure 2). Immunohistochemical staining revealed high expression of CD3, CD4>CD8, and CD163 in the infiltrate. Associated PD-L1 expression, as well as scarce Foxp $3^{+}$cells, was observed.

The patient was initially treated with high-dose potency topical steroids and rapidly moved to prednisone $1 \mathrm{mg}$ / $\mathrm{kg}$ when he did not have an adequate response. He had a mild initial good response, but the lesions flared on the taper of steroids at the same time as a grade 2 immunerelated colitis. On increase of corticosteroids, the patient had a resolution of his colitis and stability of his lesions; prednisone was tapered for a total of 2 months of treatment. He was subsequently serially treated with methotrexate for 4 weeks, followed by mycophenolate mofetil for 4 weeks, and then cyclosporine for 4 weeks, with a mild response to therapy at best. His lesions remained symptomatic, with significant discomfort and pruritus (figure 1).

After consultation in a multidisciplinary immunetoxicity team, it was decided that NBUVB phototherapy could be considered. Of interest, our patient also experienced immune-related uveitis before the start of phototherapy, which was treated with corticosteroid eye drops. The patient received 17 total sessions, which induced a significant healing of his lesions and the resolution of the pruritus and skin discomfort. Subsequent to his immunetoxicities, a significant systemic antitumor response was noted on his staging scans, which are currently ongoing over 12 months after cessation of PD-1 therapy.

\section{DISCUSSION}

Immune-related lichenoid eruptions constitute up to $25 \%$ of the cutaneous adverse events on ICIs. ${ }^{5}$ These have similar features to lichen planus and may present with flat-topped erythematous-to-violaceous scaly papules, oral or genital ulcers, leukoplakia, hypertrophic plaques, and nail dystrophy. These reactions may present with a delayed onset and even after discontinuation of ICIs, ${ }^{6}$ as was the case with our patient. An exacerbation of pre-existing mucocutaneous disease may also be observed, such as our patient's known lichen planus (oral). The predominant histological feature is lichenoid interface changes, with a CD3-positive infiltrate with a high CD4:CD8 ratio. ${ }^{26}$ Our
A.

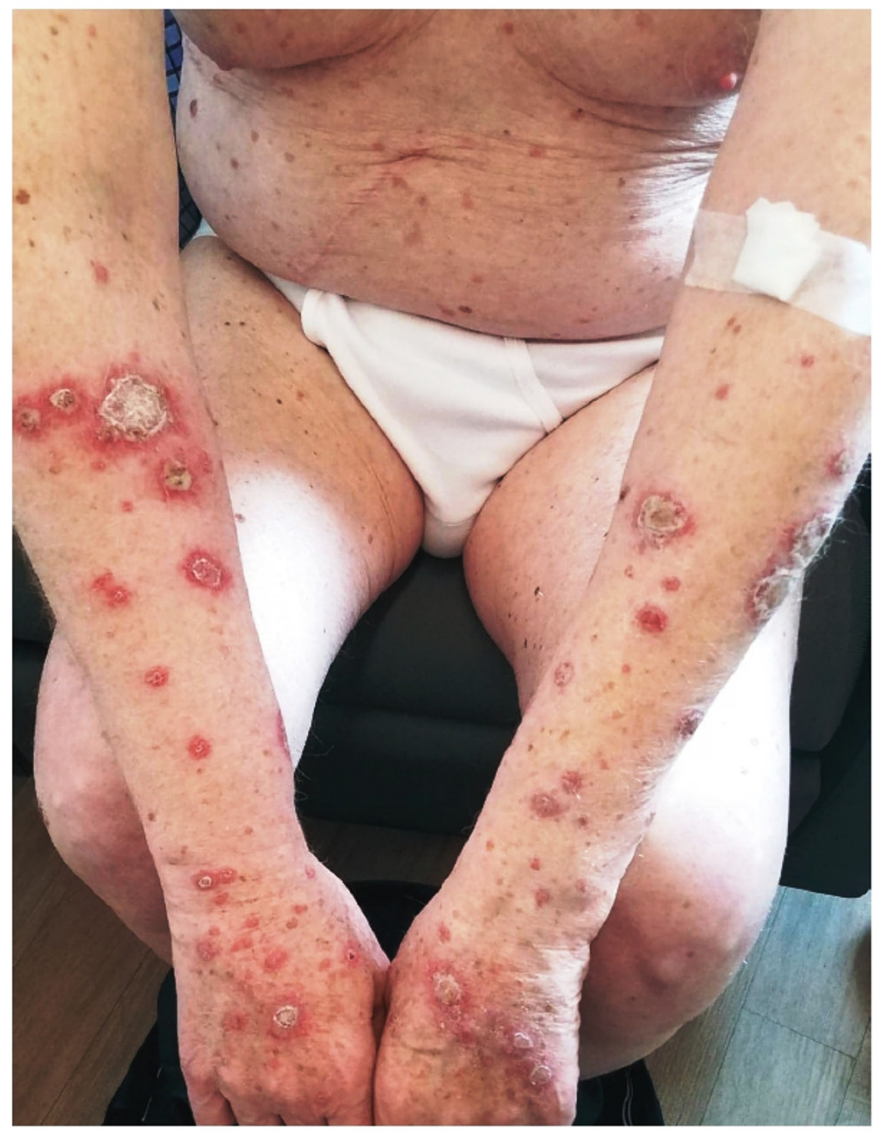

B.

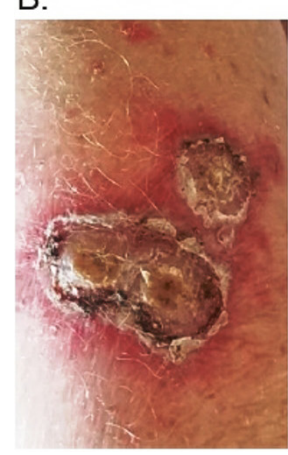

C.

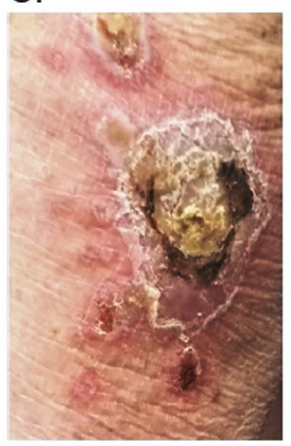

D.

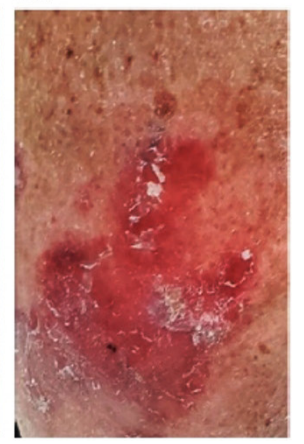

Figure 1 (A) Onset of immune-related lichenoid reaction on programmed cell death 1 therapy. The patient had multiple erythematous papules and plaques with central hyperkeratosis on the arms, trunk and upper legs, associated with significant discomfort and pruritus. (B) Magnified view of the lesions. (C) Minimal response after multiple lines of immunosuppression, including high-potency topical and oral corticosteroids, mycophenolate mofetil, methotrexate and cyclosporine. (D) Significant clinical response after 17 sessions of narrowband ultraviolet B therapy, resulting in healing of the patient's lesions and resolution of associated clinical symptoms.

immunohistochemical analyses support that lichenoid eruptions associated with PD-1 inhibitors are largely driven by $\mathrm{T}$ cells with macrophage participation. Furthermore, ICIs can shift antigen-induced reactivity towards an inflammatory Th1/Th17 response, the same response 

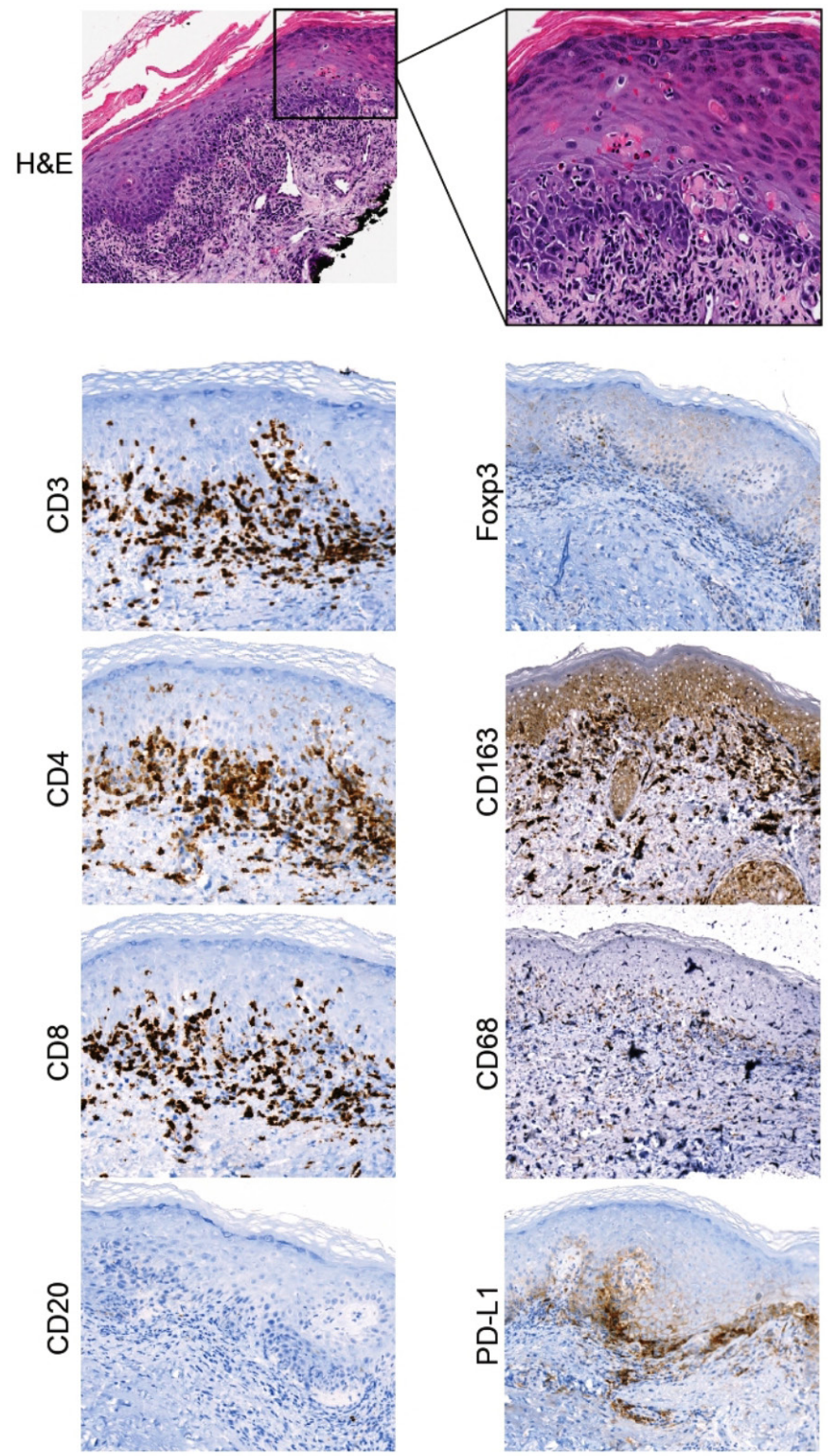

Figure 2 H\&E-stained section of the right arm lesion showed a lichenoid interface dermatitis. On higher power, exocytosis of lymphocytes and eosinophils can be seen. There are clusters of dyskeratotic cells (colloid bodies) along the basal layer and single dyskeratotic keratinocytes higher up in the epidermis. Immunohistochemical examination revealed that the lichenoid infiltrate was composed mainly of $\mathrm{T}$ cells, with a predominance of $\mathrm{CD} 4^{+}$cells; $\mathrm{CD} 8^{+} \mathrm{T}$ cells were less abundant. CD163 ${ }^{+}$histiocytes and scarce Foxp3 ${ }^{+}$ regulatory $T$ cells were also seen in the infiltrate. Staining for CD20 was negative. PD-L1 was expressed by the infiltrate. PD-L1, programmed cell death ligand 1.

which has also been observed with lichen planus. ${ }^{7}$ Therapeutic NBUVB suppresses many cell-mediated immune responses and induces a shift from a Th1/Th17 to a Th2 milieu in both preclinical and clinical models of inflammatory skin diseases. ${ }^{8}$

NBUVB phototherapy for immune-related lichenoid dermatitis has been reported as a first-line therapy in four patients, ${ }^{26}$ as well as in one patient who failed a steroid taper, ${ }^{9}$ but not for refractory cases. Our case also differs in that our patient responded to NBUVB as monotherapy, whereas the previously described patient concomitantly received systemic steroids. ${ }^{9}$ Our report therefore strengthens the evidence for effectiveness of NBUVB in this setting. In addition, our case is of interest, given a good response to this therapy after failure of multiple systemic lines of immunosuppression, including corticosteroids, methotrexate, mycophenolate mofetil, and cyclosporine. Due to patient preference, NBUVB was not trialed sooner, which could have spared him exposure to the other immunosuppressants. Also, although use of topical calcineurin inhibitors has been previously reported, they have not been showed to be superior to high-potency topical corticosteroids. ${ }^{10}$

For lichen planus, an average of 18-23 sessions of NBUVB, fractionated over three times per week, is necessary to induce an objective response. As with our case, we would therefore recommend a trial of at least 17 sessions of NBUVB three times weekly ( 6 weeks) before assessing treatment efficacy. Short-term side effects may include erythema, xerosis associated with pruritus, and occasional blistering. To date, no study has found a significant association between NBUVB therapy and a heightened risk of developing cutaneous squamous cell carcinoma, basal cell carcinoma, or melanoma.

Immune-related skin reactions are often associated with several favorable outcomes, including overall response rate, progression-free survival, and overall survival. This was the case with our patient, who achieved a near complete remission of his metastatic melanoma 12 months after pembrolizumab was discontinued.

\section{CONCLUSION}

With the increasing use of ICIs in clinical practice, a significant rise in immune-related cutaneous adverse events has emerged. Although most cases can be successfully managed with topical high-potency or oral corticosteroids, the refractory cases pose a clinical challenge. Our case supports further exploration of NBUVB phototherapy in this setting, given the relative long track record of safety and efficacy of this approach for primary models of inflammatory skin diseases.

Contributors $\mathrm{KE}$ and $\mathrm{M}-\mathrm{HHB}$ contributed equally to this work and are cofirst authors. KE, M-HHB, and $\mathrm{HZ}$ analyzed and interpreted the patient case, and wrote and edited the manuscript. KE and RB were the main treating physicians. $\mathrm{KP}$ reviewed the manuscript. MR performed the pathological interpretation and provided the histopathology figure. WHM and OR supervised this case report and reviewed the manuscript. All authors have read and approved the final manuscript.

Funding The authors have not declared a specific grant for this research from any funding agency in the public, commercial or not-for-profit sectors.

Competing interests None declared.

Patient consent for publication Not required.

Provenance and peer review Not commissioned; externally peer reviewed.

Open access This is an open access article distributed in accordance with the Creative Commons Attribution Non Commercial (CC BY-NC 4.0) license, which permits others to distribute, remix, adapt, build upon this work non-commercially, 
and license their derivative works on different terms, provided the original work is properly cited, appropriate credit is given, any changes made indicated, and the use is non-commercial. See http://creativecommons.org/licenses/by-nc/4.0/.

\section{ORCID iD}

Meagan-Helen Henderson Berg http://orcid.org/0000-0002-4622-2931

\section{REFERENCES}

1 Hodi FS, Chiarion-Sileni V, Gonzalez R, et al. Nivolumab plus ipilimumab or nivolumab alone versus ipilimumab alone in advanced melanoma (CheckMate 067): 4-year outcomes of a multicentre, randomised, phase 3 trial. Lancet Oncol 2018;19:1480-92.

2 Coleman E, Ko C, Dai F, et al. Inflammatory eruptions associated with immune checkpoint inhibitor therapy: a single-institution retrospective analysis with stratification of reactions by toxicity and implications for management. J Am Acad Dermatol 2019;80:990-7.

3 Esfahani K, Elkrief A, Calabrese C, et al. Moving towards personalized treatments of immune-related adverse events. Nat Rev Clin Oncol 2020;17:504-15.
4 Sigmundsdottir $\mathrm{H}$, Johnston A, Gudjonsson JE, et al. Narrowband-UVB irradiation decreases the production of proinflammatory cytokines by stimulated T cells. Arch Dermatol Res 2005;297:39-42.

5 Hwang SJE, Carlos G, Wakade D, et al. Cutaneous adverse events (AEs) of anti-programmed cell death (PD)-1 therapy in patients with metastatic melanoma: A single-institution cohort. J Am Acad Dermatol 2016;74:455-61.

6 Shi VJ, Rodic N, Gettinger S, et al. Clinical and histologic features of lichenoid mucocutaneous eruptions due to Anti-Programmed cell death 1 and Anti-Programmed cell death ligand 1 immunotherapy. JAMA Dermatol 2016;152:1128-36.

7 Solimani F, Pollmann R, Schmidt T, et al. Therapeutic targeting of Th17/Tc17 cells leads to clinical improvement of lichen planus. Front Immunol 2019;10:10.

8 Weichenthal M, Schwarz T. Phototherapy: how does UV work? Photodermatol Photoimmunol Photomed 2005;21:260-6.

9 Donaldson M, Owen JL, Chae YK, et al. Management of persistent pruritus and lichenoid reaction secondary to nivolumab with Narrowband ultraviolet B phototherapy. Front Oncol 2018;8:405.

10 El-Batawy MMY, Bosseila MA-W, Mashaly HM, et al. Topical calcineurin inhibitors in atopic dermatitis: a systematic review and meta-analysis. J Dermatol Sci 2009;54:76-87. 